\title{
Unmanned ground vehicle positioning system by GPS/dead-reckoning/IMU sensor fusion
}

\author{
Meng Zhang ${ }^{\dagger}$ and Ke Liu \\ State Grid Shandong Electric Power Research Institute, \\ Jinan, Shandong 250003, P. R. China \\ E-mail: mengzhangxjtu@126.com \\ Chen $\mathrm{Li}$ \\ State Grid Qihe Power Supply Company, \\ Qihe, Shandong 251100, P. R. China
}

\begin{abstract}
Real-time positioning system is critical for control and navigation of unmanned ground vehicles. In this paper, we present a low-cost integrated GPS/DR/IMU positioning solution. A two-level adaptive Kalman Filter based algorithm is introduced to fuse sensor signals. Experimental results demonstrate a much better performance with accurate and robustness output even during short-time GPS signal drop-out.
\end{abstract}

Keywords: Unmanned Vehicle; Kalman Filter; GPS/IMU; DR.

\section{Introduction}

In recent years, there are extensive researches and related applications on unmanned ground vehicles or mobile robots. E.g., Google has proceeded its unmanned vehicle projects for years and Tesla has released electrical cars with self-driving mode. The positioning systems, which provide real-time position and attitude information, are key components to enhance vehicular control and navigation performance.

There are two main categories for positioning systems: absolute and relative measurements. GPS, whose measurement calculation is based on the pseudoranges sent from different satellites, is one of the most widely used absolute positioning systems. It can provide three-dimension position and velocity information on a global scale with no cumulative errors and obtain consistent precision over time. One major hurdle to GPS inherent in its method of operation is interference or blockage of satellite reception, especially in urban areas where exist lots of trees, buildings, etc. Moreover, GPS outputs frequency is low, the position and velocity data usually has random noise. 
The Inertial Navigation System(INS) and Dead-Reckoning System(DRS) are common systems for relative positioning. INS uses gyroscopes and accelerometers outputs to maintain relative pose information. DRS is specially used for land wheeled vehicles, which uses speed output from an odometer and heading output from a gyroscope for position prediction. The output of INS or DRS is highly autonomous, almost free from external interference, with good smoothness and short-time accuracy. The major drawback for relative positioning is that the measurement is based on integration of data along traveling course. Errors would accumulate by time and tend to unbounded.

Unmanned vehicles require consistent high precision positioning information free from external interference, which is impossible to provide by any single positioning system. GPS and autonomous positioning systems have good complementarities, and sensor fusion has become a hot spots for real-time ground positioning researches. Sun[1] introduced an on-board GPS/MEMS IMU system using low-cost MEMS fabricated INS to enhance GPS performance. Yang[2] focus on a complete GPS/INS integration using discrete Kalman Filter and Fuzzy Logic. Fouque [3] implemented an map-aided odometer Deadreckoning GNSS-aided precise localization system with pre-collected map information. Malyavej [4] discussed a GPS/DR fusion approach using Unscented Kalman Filter (UKF). Elkaim [5] built a practical test method for the performance of two different low-cost GPS/INS commercial products with Differential GPS receiver and high precision KVH FOG. In terms of sensors picked-up, the common combination would be: GPS/high précised-INS, GPS/low-cost IMU, GPS/DR, etc. While in terms of fusion methods, the Kalman Filter and its applications, e.g., Extended Kalman Filter (EKF) [6], UKF [4], Federal Kalman Filter (FKF) [7] are mostly used. The other methods, e.g., Neural Network, Fuzzy Fusion are also been discussed by some researchers.

In this paper, we introduce a GPS/DR/IMU sensor fusion system, which combines measurement from a GPS receiver, an onboard odometer, a Fiber Gyroscope, and a low-cost MEMS IMU. A distributed strategy and several lowdimensional adaptive Kalman Filters would be introduced instead of using high dimensional EKF, UKF, etc. The computational time would be saved and the outputs robustness would be guaranteed. By unique sensors combination and appropriate algorithm design, the fusion system provides high-frequency, highprecision and robust pose outputs comparable to expensive GPS/INS systems in approximate 2D plane urban environments.

The paper is organized as follows. Section 2 introduce system hardware and software layout. Section 3 introduce sensor signal parsing and pre-filtering principle. Section 4 introduce the fusion method and experimental results. Section 5 is the final conclusions. 


\section{HW/SW Layout}

The system organization is shown in Fig 1, the real-time information from GPS and other sensors is transmitted to embedded computer through RS232 interface.

The raw data will be parsed and pre-filtered respectively before fusion process. The data fusion is implemented by a two-step low dimensional Kalman Filter system. The heading and speed information will be filtered respectively first, then a Position Kalman Filter is introduced to fusion GPS and the filtered heading/speed information. Finally, the positionlspeedlheading information is obtained and sent to vehicles command platform through UDP packets.

Real-time

Position \speed $\backslash$ heading

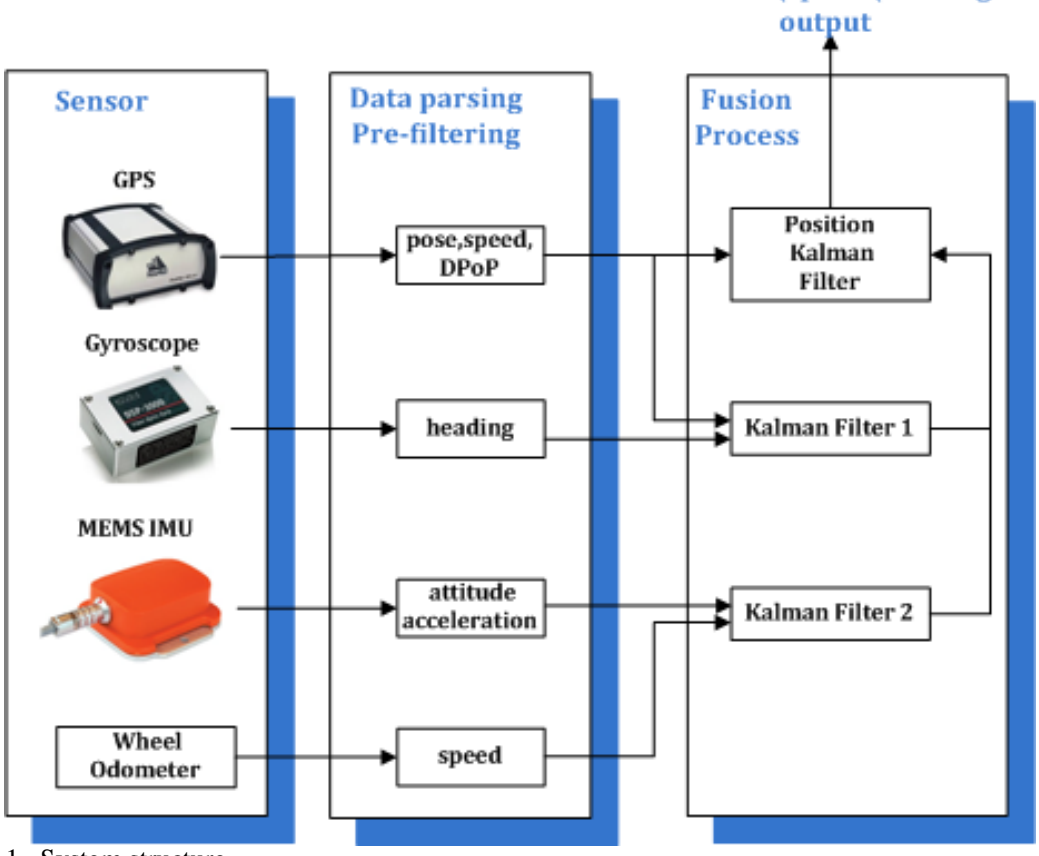

Fig. 1. System structure

\section{Data Parsing and Pre-filtering}

\section{1. $G P S$}

The ProPak-G2 NovAtel GPS can output 20HZ real-time three-dimensional position, velocity, and Position-Dilution of Precision (PDoP) [8] information in WGS84 global frame. In the approximate 2D plane area, the tangent East-NorthUp (ENU) frame is usually introduced for ground positioning. Then, by choosing a reference point, the transformation between WGS84 and ENU is computed [9]. 
PDoP is an important factor that indicates the accuracy of GPS positioning. The smaller the value of PDoP, the higher the GPS positioning accuracy. In the design of Position Kalman Filter, PDoP will be used as a key factor for error modeling.

\subsection{Gyroscope}

The KVH 3000 Fiber-Optic-Gyroscope (FOG) outputs integrated angle data in $1000 \mathrm{HZ}$, which can be used as relative heading for unmanned vehicles. The FOG is installed horizontally in the center of the vehicle. Due to angular random walk, zero-drift effects, vibration noise, ground speed interference and etc, the heading error accumulates over time. Set the vehicle lay still in a horizontal plane, turn on the onboard generator and engine at the same time(consider the noise caused by vibration), the angle outputs results from several tests is shown in Fig 2.
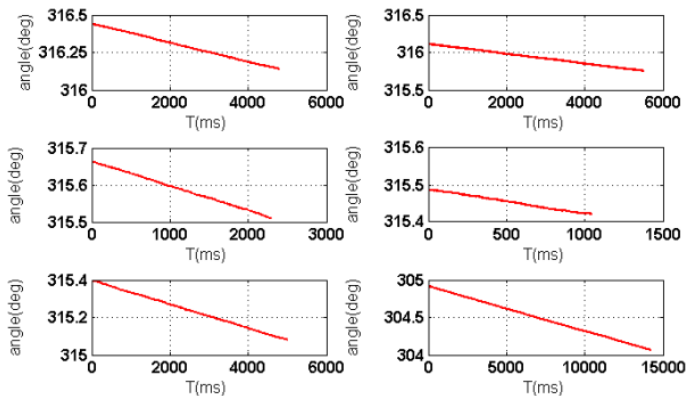

Fig. 2. Test results of KVH FOG angle output.

In static state, the cumulative error of FOG angle is approximately linearly increased. Record static angle outputs in different times, and calculate cumulative error as Table 1:

Table 1. FOG angle cumulative error statistics

\begin{tabular}{ll}
\hline Data samples & Cumulative error(deg/s) \\
\hline 1 & -0.0053 \\
2 & -0.0051 \\
3 & -0.0049 \\
4 & -0.0050 \\
5 & -0.0047 \\
6 & -0.0048 \\
\hline
\end{tabular}

Take average of the data above, we get a error compensation value for heading angle output $\delta=-0.050 \mathrm{deg} / \mathrm{s}$. By using this simple compensation 
method, the error can be partially eliminated. The heading output error can be controlled within $5 \mathrm{deg} / \mathrm{hr}$.

\subsection{MEMS IMU}

The Mti MEMS IMU can output 3D position, altitude and acceleration data in $50 \mathrm{~Hz}$. The acceleration in horizontal direction will be used to fuse odometer speed and the pitch/roll will be used for vehicle control system.

\subsection{Odometer}

The odometer speed information is obtained from vehicle CAN bus directly, with a output rate of $10 \mathrm{~Hz}$. We use a Differential GPS module to calibrate odometer output to acquire better accuracy. The calibration test should be held in open and flat ground environment, and make the vehicle moves in a straight line. Note that there exists slipping and relative friction between wheels and ground, and the degree of friction and slipping is different in acceleration and deceleration mode. The odometer must apply different calibration coefficient in respective moving state.

\section{Fusion Method and Experimental Results}

\subsection{Speed Kalman Filter}

Odometer works poor in inflated or slippery ground and in low speed moving mode. A two dimensional EKF method is applied to combine MEMS IMU acceleration with odometer speed by using constant acceleration model. The fused speed has a better performance in low-speed or poor ground conditions.

\subsubsection{The state and measurement equation}

The discrete state vector and equation is shown in Eq. (1). (2).

$$
\begin{aligned}
& X_{k}=\left[\begin{array}{ll}
v_{k} & a_{k}
\end{array}\right]^{T} . \\
& X_{k+1}=\mathrm{AX}_{k}+W_{k} .
\end{aligned}
$$

Where $v_{k}, a_{k}$ represents speed and acceleration, $\mathrm{A}$ is the transfer matrix and $W_{k}$ is the process noise. Assume the sampling period is $t$, and max change of acceleration is $\sigma$, the process noise would be:

$$
W_{k}=\left[\begin{array}{l}
t \\
1
\end{array}\right] \sigma
$$


The process covariance noise matrix $\mathrm{Q}$ can be calculated as:

$$
Q=E\left(W_{k} W_{k}^{T}\right)=\left[\begin{array}{ll}
t^{2} & t \\
t & 1
\end{array}\right] \sigma^{2}
$$

The measurement equation is shown in Eq. (5).

$$
Z_{k}=H X_{k}+V_{k} \text {. }
$$

Where, $Z_{k}=\left[\begin{array}{ll}\tilde{v}_{k} & \tilde{a}_{k}\end{array}\right]^{T}$ represents the speed and acceleration measurement vector, $H=I_{2}$ represents second order unit matrix, and $V_{k}=\left[\begin{array}{ll}v_{v} & v_{a}\end{array}\right]^{T}$ is the measurement noise vector of speed and acceleration.

\subsubsection{Parameter determination}

Using the odometer sampling rate $10 \mathrm{~Hz}$ as the update frequency, the covariance matrix value is obtained:

$$
Q=\left[\begin{array}{cc}
0.0025 & 0.025 \\
0.025 & 0.25
\end{array}\right]
$$

The measurement noise is evaluated from sensor output error by using DGPS as standard reference. Through various test results, the measurement covariance noise matrix in different speed interval is :

$$
R_{l}=\left[\begin{array}{cc}
0.0441 & 0.011 \\
0.011 & 0.0027
\end{array}\right], R_{n}=\left[\begin{array}{ll}
0.0031 & 0.0029 \\
0.0029 & 0.0027
\end{array}\right], R_{h}=\left[\begin{array}{cc}
0.070 & 0.013 \\
0.013 & 0.0027
\end{array}\right]
$$

Where $R_{l} . R_{n}, R_{h}$ represent matrix value in low-speed $(0 \sim 4 \mathrm{~m} / \mathrm{s})$, normal $\operatorname{speed}(4 \sim 10 \mathrm{~m} / \mathrm{s})$ and high speed( $>10 \mathrm{~m} / \mathrm{s})$.

\subsubsection{Experimental results}

Use DGPS as the standard speed reference, the odometer speed error and the fusion speed error comparison results are shown in Fig 3. Obviously, the speed output obtain better performance after sensor fusion. 

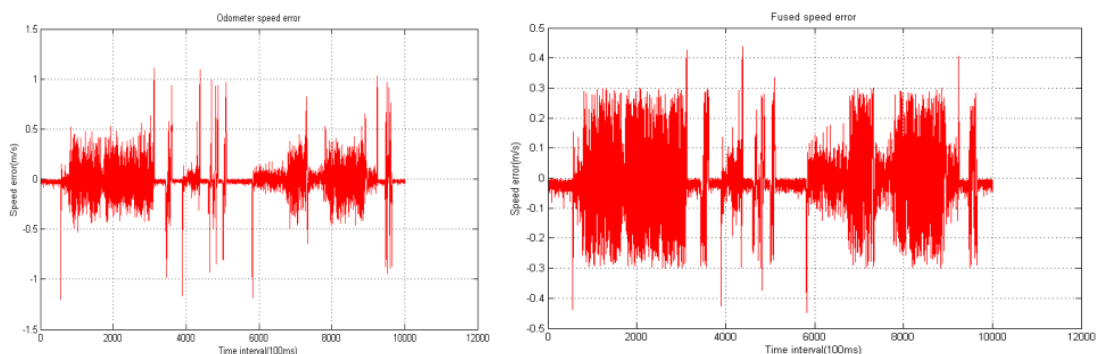

Fig. 3. Test results of speed error from odometer and odometer/MEMS fusion

\subsection{Heading Kalman Filter}

The heading estimation from GPS receiver can only be used in dynamic situations. It is combined with random noise and is susceptible to environmental interference. The relative heading from FOG is stable and precise in short term, while it has cumulative error, needs to be initialized by GPS for global positioning use. Heading Kalman Filter is designed to integrate the heading output of the two systems, and obtain heading with precision and robustness in both dynamic and static conditions.

\subsubsection{Filter design}

Using the FOG heading error as the estimator, an Indirect Kalman Filter (IKF)model is introduced. The state and measurement equations is shown as below:

$$
\begin{gathered}
\delta \theta_{k}=\delta \theta_{k-1}+\delta_{d r i f t}+\delta_{\text {noise }} \\
Z(\delta \theta)=\theta_{F O G}-\theta_{g p s}=\delta \theta_{F O G}-\delta \theta_{g p s}=\delta \theta+v
\end{gathered}
$$

Where $\delta_{\text {drift }}$ and $\delta_{\text {noise }}$ represent drift and random error of FOG respectively, . $\delta \theta$ represents FOG heading error and $v$ represents GPS measurement noise.

The real-time IKF process is shown in Fig 4 


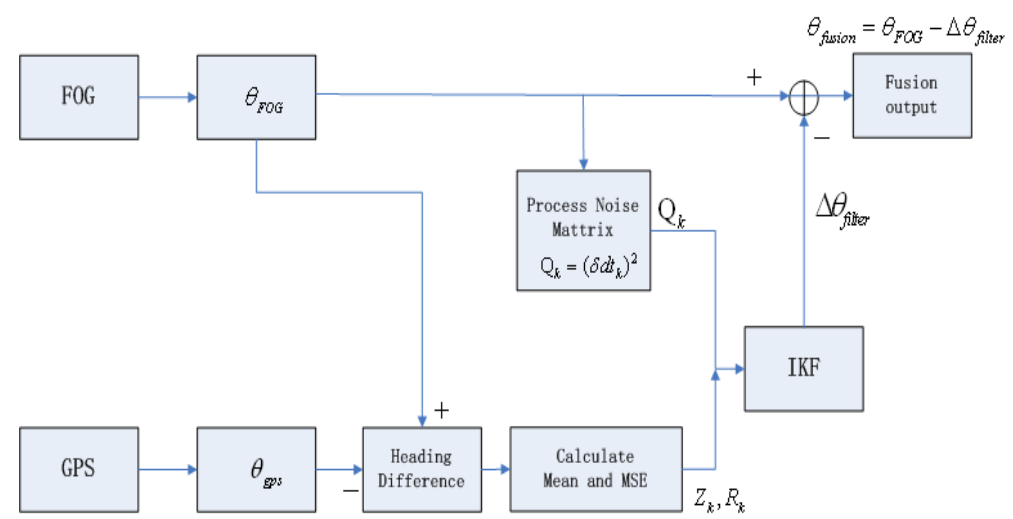

Fig. 4. IKF fusion process for FOG and GPS heading output .

\subsubsection{Experimental results}

After filter initialization in open ground, the vehicle use urban area as testing environment, comparison results is shown in Fig. 5.

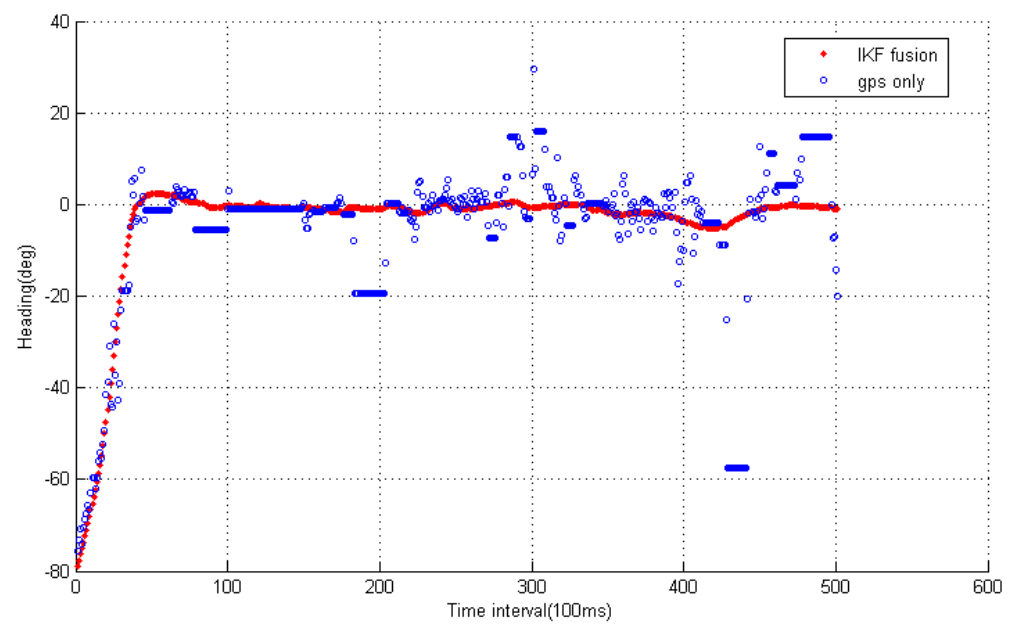

Fig. 5. IKF comparison of heading output (GPS only and IKF Fusion) .

\subsection{Position Kalman Filter}

GPS is sensitive to signal dropout and hostile jamming, a position Kalman Filter which fuses GPS with local positioning measurement is introduced to reduce noise and retain position/attitude during signal drop-out. 


\subsubsection{Filter design}

Suppose the unmanned vehicle moves in approximate 2D plane, the prediction process is modeled by Dead-Reckoning algorithm, the initialization and measurement output of KF is obtained from GPS signal analysis. The discrete state and measurement equations is:

$$
\begin{gathered}
{\left[\begin{array}{l}
x_{e}(k) \\
x_{n}(k)
\end{array}\right]=\left[\begin{array}{ll}
1 & 0 \\
0 & 1
\end{array}\right]\left[\begin{array}{l}
x_{e}(k-1) \\
x_{n}(k-1)
\end{array}\right]+\left[\begin{array}{c}
T \cos \theta \\
T \sin \theta
\end{array}\right] V+\left[\begin{array}{l}
w_{e} \\
w_{n}
\end{array}\right]} \\
{\left[\begin{array}{l}
\mathrm{z}_{e}(k) \\
z_{n}(k)
\end{array}\right]=\left[\begin{array}{ll}
1 & 0 \\
0 & 1
\end{array}\right]\left[\begin{array}{l}
x_{e}(k) \\
x_{n}(k)
\end{array}\right]+\left[\begin{array}{l}
v_{e}(k) \\
v_{n}(k)
\end{array}\right]}
\end{gathered}
$$

The general filter process is shown in Fig 6 .

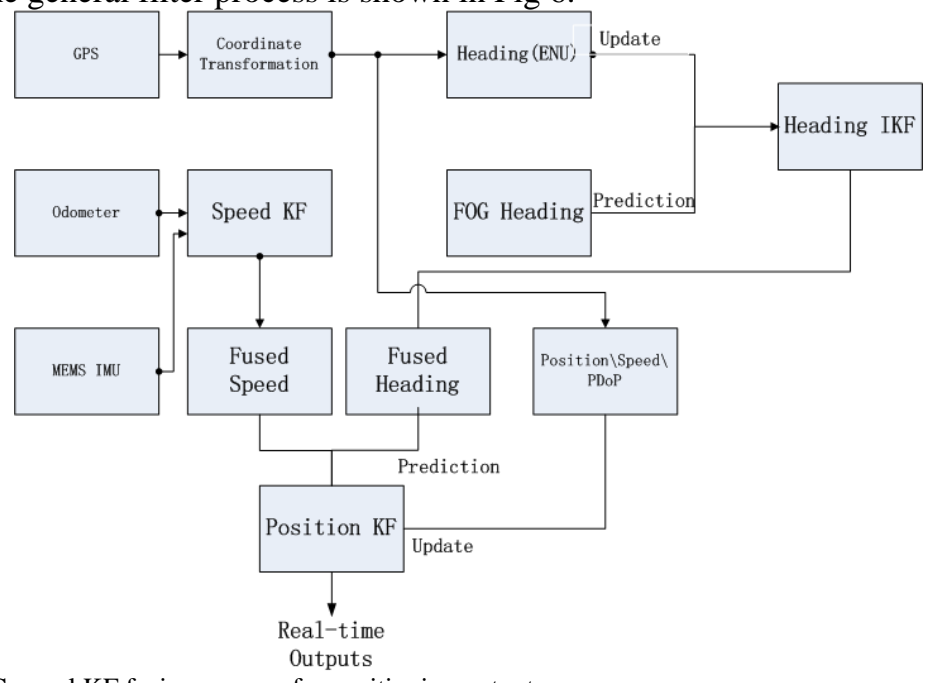

Fig. 6. General KF fusion process for positioning output .

\subsubsection{Experimental results}

Test route is selected from city environments, the vehicle started from open ground for initialization, then passed through urban roads with many trees and buildings, and finally entered indoor parking lot where satellite signals were totally blocked.The output of fusion data can well represent the performance of filter in this complex and multi-interference environment, shown in Fig 7. 


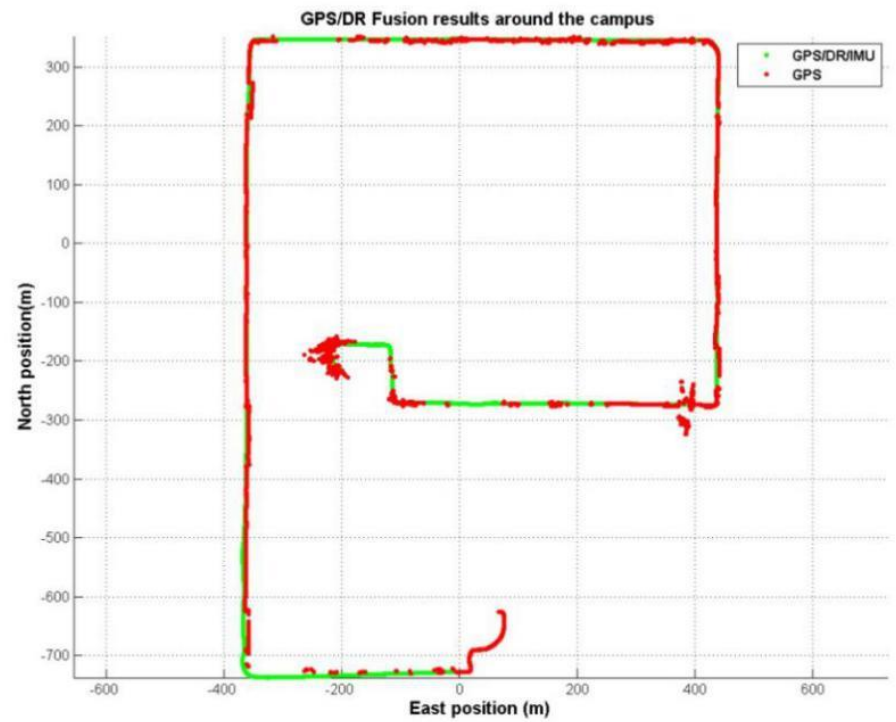

Fig. 7. General KF fusion process for positioning output

\section{Conclusions}

This paper presents fusion algorithm and implementation of positioning system A two-step discrete KF system using signals from GPS and other local positioning sensors is introduced. Experimental results demonstrate that the KF based algorithm can improve accuracy, robustness and retain positioning output during short-term of GPS signal drop-out. The fusion system presented in this paper offer a practical exploration for unmanned vehicle navigation with adequate performance and affordable cost for commercialization.

\section{References}

1. CJ. Sun, C. Hung, et al, Implementation of GPS/INS navigation System using Low-Cost MEMS Sensors, the 5th IEEE Conference on Industrial Electronics and Applications, 2010, pp. 1588-1592.

2. QM. Yang, JM. Sun, A Location Method for Autonomous Vehicle Based on Integrated GPS/INS, IEEE International Conference on Vehicular Electronics and Safety, Beijing, 2007.

3. C. Fouque, P. Bonnifait, et al, Enhancement of Global Vehicle Localization using Navigable Road Maps and Dead-Reckoning, Position, Location \& Navigation Symposium, IEEE/ION, 2008, pp. 1286-1291.

4. V. Malyavej, P. Torteeka, Unmanned Ground Vehicle Localization by Dead-reckoning/GPS sensor fusion, International Conference on Electrical 
Engineering/Electronics Computer Telecommunications and Information Technology, 2010, pp. 508-512.

5. G. H. Elkaim, M. Lozarraga, et al, Comparison of Low-Cost GPS/INS Sensors for Autonomous Vehicle Applications, Position, Location and Navigation Symposium, IEEE/ION , 2008, pp. 1133-1144.

6. B. Li, L. Cai, MQ. X, Adaptive extended Kalman filtering algorithm for SINS/GPS integrated navigation in guided munitions, IEEE International Conference on Intelligent Computing and Intelligent Systems, 2010, 2: pp. 283-287.

7. NA. Carlson. Federated filter for fault-tolerant integrated navigation systems, IEEE Position Location and Navigation Symposium, 1988, pp. 110-119.

8. HH Wang, XQ Zhan, et al, Geometric dilution of precision for GPS singlepoint positioning based on four satellites, Systems Engineering and Electronics, 2008, 19(5), pp. 1058-1063.

9. E.D. Kaplan, Understanding GPS: principles and applications, A. House, Ed Boston, 1996. 